H. Matsumura

Nagoya Math. J.

Vol. 87 (1982), 227-245

\title{
INTEGRABLE DERIVATIONS
}

\author{
HIDEYUKI MATSUMURA \\ Dedicated to Prof. Yoshikazu Nakai on his Sixtieth Birthday
}

\section{Introduction}

Let $A$ be a commutative ring and $D$ be a derivation of $A$ into itself. If there exists a homomorphism $E: A \rightarrow A[[t]]$ such that

$$
E(a) \equiv a+t D(a) \quad \bmod t^{2}
$$

then we say that $D$ is integrable. Integrable derivations have many good properties. In fact, most of unpleasant phenomena of derivations in characteristic $p$ disappear if we consider integrable derivations only.

In $\S 1$ we state definitions and basic properties of differentiations, and we give some examples of non-integrable derivations.

$\S 2$ is devoted to theorems which are essentially due to Seidenberg ([18], [19], [20]). These theorems show that integrable derivations behave as they should, and provides us with necessary conditions for integrability.

Then in $\S 3$ and $\S 4$ we prove some sufficient conditions. In $\S 3$ we consider smooth or formally smooth algebras, using André's homology theory. In $\S 4$, by an elementary argument we prove a criterion of integrability, which shows that there are plenty of integrable derivations (in the case of an integral domain finitely generated over a perfect field).

\section{$\S 1$. Definitions and examples}

In this article all rings are assumed to be commutative with a unit element. Local rings are assumed to be noetherian.

Let $A$ be a ring. The set of all derivations of $A$ into itself is an $A$-module and is denoted by $\operatorname{Der}(A)$. If $k$ is a subring of $A$, the submodule of $\operatorname{Der}(A)$ consisting of those derivations which vanish on $k$ is denoted by $\operatorname{Der}_{k}(A)$.

Received December 26, 1980. 
A differentiation $\underline{D}$ of $A$ (in the sense of Hasse-Schmidt [7]) is an infinite sequence $\underline{D}=\left(D_{0}, D_{1}, D_{2}, \cdots\right)$ of additive endomorphisms $D_{i}: A \rightarrow$ $A$ such that

$$
D_{0}=\text { identity }, \quad D_{n}(a b)=\sum_{i+j=n} D_{i}(a) D_{j}(b) .
$$

It follows that $D_{1}$ is a derivation. $D_{i}$ will be called the $i$-th component of $\underline{D}$. Let $t$ be an indeterminate over $A$, and put

$$
E(a)=E_{t}(a)=\sum_{n=0}^{\infty} t^{n} D_{n}(a) \in A[[t]] \quad(a \in A) .
$$

Then $E=E_{t}$ is a ring homomorphism from $A$ into $A[[t]]$ such that $a \equiv$ $E(a) \bmod t$. It can be uniquely extended to an endomorphism of $A[[t]]$ such that $E(t)=t$; namely, we define

$$
E\left(\sum_{i=1}^{\infty} t^{i} a_{i}\right)=\sum_{i=0}^{\infty} t^{i} E\left(a_{i}\right)
$$

Then, using

$$
E(a) \equiv a \bmod t(a \in A), \quad E(t)=t,
$$

we can easily see that $E$ is an automorphism of $A[[t]]$. Conversely, any automorphism of $A[[t]]$ satisfying (1.4) comes from a differentiation. We will denote the automorphism $E=E_{t}$ obtained from $\underline{D}$ by $\Lambda(\underline{D})$; thus $\Lambda$ is a bijection from the set of differentiations of $A$ to the set of automorphisms of $A[[t]]$ satisfying (1.4). This latter set is obviously a subgroup of Aut $(A[[t]])$, therefore by means of $\Lambda$ we can make the set of differentiations a group, which we denote by $\operatorname{HS}(A)$ and call the Hasse-Schmidt group of $A$. If $\underline{D}=\left(1, D_{1}, \cdots\right)$ and $\underline{D}^{\prime}=\left(1, D_{1}^{\prime}, \cdots\right)$ are differentiations of $A$, easy calculations show that

$$
\begin{aligned}
& \underline{D} \underline{D}^{\prime}=\left(1, D_{1}+D_{1}^{\prime}, D_{2}+D_{1} D_{1}^{\prime}+D_{2}^{\prime}, \cdots, \sum_{i} D_{i} D_{n-i}^{\prime}, \cdots\right) \text { and } \\
& \underline{D}^{-1}=\left(1,-D_{1}, D_{1}^{2}-D_{2},-D_{1}^{3}+D_{1} D_{2}+D_{2} D_{1}-D_{3}, \cdots\right) .
\end{aligned}
$$

Moreover, from (1.1) we see that

(1.7) if $x \in A$, then $\left(1, x D_{1}, x^{2} D_{2}, \cdots, x^{n} D_{n}, \cdots\right)$ is a differentiation.

We say that a derivation $D \in \operatorname{Der}(A)$ is integrable if there exists a differentiation $\underline{D}=\left(1, D_{1}, D_{2}, \cdots\right)$ of $A$ with $D_{1}=D$. Such $\underline{D}$ is called (by lack of better terminology) an integral of $D$; we also say that $\underline{D}$ lifts $D$. The formulas (1.5), (1.6), (1.7) show that the set of integrable derivations 
of $A$ is an $A$-submodule of $\operatorname{Der}(A)$. We denote it by $\operatorname{Ider}(A)$. If $A$ contains the rational number field $\mathbf{Q}$ it is easy to see that all derivations are integrable. The same holds when $A$ is a field (of any characteristic), see Th. 6. But in general there are non-integrable derivations.

Remark 1. If $t^{\prime}$ is an element of $A[[t]]$ without constant term and $\underline{D}$ is a differentiation of $A$, we get a homomorphism $E_{t^{\prime}}: A \rightarrow A[[t]]$ by $E_{t^{\prime}}(a)$ $=\sum_{n=0}^{\infty} t^{\prime n} D_{n}(a)$, and this can be uniquely extended to an automorphism of $A[[t]]$ as in (1.3). Applying $\Lambda^{-1}$ to this, we get a new differentiation. For instance if $t^{\prime}=x t$ then we get the differentiation of (1.7).

Remark 2. If $E_{t}$ and $E_{t}^{\prime}$ correspond to $\underline{D}$ and $\underline{D}^{\prime}$ respectively, and if we put $s=t^{n}$ for some $n>1$, then $E_{t} \circ E_{s}^{\prime}$ gives a differentiation of the form $\left(1, D_{1}, \cdots, D_{n-1}, D_{n}+D_{1}^{\prime}, \cdots\right)$. From this it is clear that an integrable derivation can have many integrals.

Let $k$ be a subring of $A$. A differentiation $\underline{D}=\left(1, D_{1}, D_{2}, \cdots\right)$ is called a differentiation of $A$ over $k$ if $D_{i}(a)=0$ for all $i>0$ and for all $a \in k$. The set of such differentiations is denoted by $\operatorname{HS}_{k}(A)$. A derivation $D$ is said to be integrable over $k$ if it has an integral belonging to $\operatorname{HS}_{k}(A)$. The set of derivations which are integrable over $k$ will be denoted by $\operatorname{Ider}_{k}(A)$, which should not be confused with $\operatorname{Der}_{k}(A) \cap \operatorname{Ider}(A)$. For instance, if $A$ is a ring of characteristic $p$ and if $k=A^{p}$, then we have $\operatorname{Der}(A)=\operatorname{Der}_{k}(A)$, but in most cases $\operatorname{Ider}(A)$ is not equal to $\operatorname{Ider}_{k}(A)$, the latter being zero if $A$ is reduced. In fact, if $\underline{D}=\left(1, D_{1}, D_{2}, \cdots\right) \in \operatorname{HS}(A)$ and if $q=p^{r}$ is a power of $p$, then we have

$$
E_{t}\left(a^{q}\right)=E_{t}(a)^{q}=\left(a+t D_{1}(a)+\cdots\right)^{q}=a^{q}+t^{q} D_{1}(a)^{q}+\cdots,
$$

therefore it holds that

$$
D_{i}\left(a^{q}\right)=0 \text { if } i \neq \equiv 0(q), \quad D_{q}\left(a^{q}\right)=D_{1}(a)^{q} .
$$

A differentiation $\underline{D}$ is said to be iterative if

$$
D_{i} \circ D_{j}=\left(\begin{array}{c}
i+j \\
i
\end{array}\right) D_{i+j} \quad \text { for all } i, j \text {. }
$$

This is equivalent to saying that the following diagram

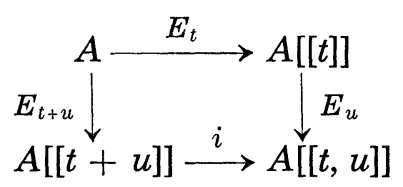


(where $i$ is the inclusion map and $\left.E_{u}(t)=t\right)$ is commutative.

A derivation $D$ will be said to be strongly integrable if it has an iterative differentiation as integral.

If the ring $A$ contains the rational number field $\mathbf{Q}$, then every derivation $D \in \operatorname{Der}(A)$ is strongly integrable, and there is a unique iterative differentiation which lifts $D$, namely $\left(1, D,(1 / 2 !) D^{2}, \cdots,(1 / n !) D^{n}, \cdots\right)$. When $A$ is of characteristic $p$, a strongly integrable derivation $D$ must satisfy $D^{p}=0$. In fact, if $\underline{D}=\left(1, D_{1}, D_{2}, \cdots\right)$ is iterative, then by induction we have $D_{1}^{i}=i$ ! $D_{i}$ (all $i$ ), hence $D_{1}^{p}=0$. The condition $D^{p}=0$ is also sufficient for strong integrability when $A$ is a field (cf. Th. 7). In the case of characteristic $p$ the strongly integrable derivations do not form an $A$ module.

We shall say that differentiations $\underline{D}=\left(1, D_{1}, D_{2}, \cdots\right)$ and $\underline{D}^{\prime}=\left(1, D_{1}^{\prime}\right.$, $\left.D_{2}^{\prime}, \cdots\right)$ commute if $D_{i}$ and $D_{j}^{\prime}$ commute for every pair $(i, j)$. If $\underline{D}$ and $\underline{D}^{\prime}$ are iterative and commute with each other, then their product $\underline{D} \underline{D}^{\prime}$ is again iterative, because $\left(E_{u} E_{u}^{\prime}\right)\left(E_{t} E_{t}^{\prime}\right)=E_{u} E_{t} E_{u}^{\prime} E_{t}^{\prime}=E_{t+u} E_{t+u}^{\prime}$ (where all maps are viewed as automorphisms of $A[[t, u]]$ which leave $t, u$ invariant).

Like derivations, differentiations can be uniquely extended to a localization. In fact, let $A$ be a ring, $S$ a multiplicative subset of $A, \underline{D} \in \operatorname{HS}(A)$ and $E_{t}: A \rightarrow A[[t]]$ the homomorphism corresponding to $\underline{D}$. Let $\psi: A \rightarrow$ $A_{s}$ and $\psi^{\prime}: A[[t]] \rightarrow A_{S}[[t]]$ be the natural maps. If $s \in S$ then the element $\psi^{\prime}\left(E_{t}(s)\right)=\psi(s)+t \psi\left(D_{1}(s)+\cdots\right.$ is invertible in $A_{s}[[t]]$, whence $\psi^{\prime} \circ E_{t}$ factors through $A_{S}$, i.e. there exists a unique homomorphism $E^{\prime}: A_{S} \rightarrow$ $A_{s}[[t]]$ satisfying $\psi^{\prime} \circ E_{t}=E^{\prime} \psi \circ$.

Similarly, if $I$ is an ideal of $A$ and $A^{*}$ is the $I$-adic completion of $A$, then a differentiation $\underline{D}=\left(1, D_{1}, D_{2}, \ldots\right)$ is uniquely extended to $A^{*}$. In fact, we have $D_{n}\left(I^{\nu}\right) \subseteq I^{\nu-n}$ for $\nu>n$, and so each $D_{n}$ is uniformly continuous in the $I$-adic topology and can be uniquely extended to the completion $A^{*}$.

Example 1. Let $k$ be a ring of characteristic $p$, and put $A=k[X] /\left(X^{p}\right)$. Put $x=X \bmod X^{p}$. Define $D \in \operatorname{Der}_{k}(A)$ by $D x=1$ (thus $D$ is induced by $d / d x$ of $k[X])$. If $D$ were integrable we would have

$$
0=E_{t}\left(x^{p}\right)=E_{t}(x)^{p}=(x+t+\cdots)^{p}=t^{p}+\cdots,
$$

which is a contradiction. Therefore $D$ is not integrable. The derivation $x D$ is integrable: in fact, $x \mapsto x(1+t) \in A[[t]]$ defines a $k$-algebra homomorphism. We have $\operatorname{Der}_{k}(A)=A \cdot D$ (a free $\operatorname{module}$ ), $\operatorname{Ider}_{k}(A)=x A \cdot D \simeq$ 
$x A$ (not free).

ExAmple 2. Let $R$ be a discrete valuation ring of characteristic zero with maximal ideal $p R$, where $p$ is a prime number. Put $k=R / p R, A$ $=R[X, Y] /\left(p X-Y^{p}\right)$. Then the derivation $Y^{p-1} \partial / \partial X+\partial / \partial Y$ of $R[X, Y]$ induces a derivation $D$ of $A$, which is not integrable. In fact, $D$ induces a derivation $\bar{D}$ of $A / p A=k[X, Y] /\left(Y^{p}\right)$ such that $\bar{D}(y)=1$, and as in the preceding example $\bar{D}$ is not integrable. If $D$ were integrable then $\bar{D}$ would be so.

ExAmple 3. Let $k$ be a field of characteristic $p$, and let $A=k[x, y]$ $=k[X, Y] /\left(Y^{p}-X^{p}-X^{p+1}\right)$. The polynomial $Y^{p}-X^{p}(1+X)$ is irreducible (Eisenstein criterion), hence $A$ is an integral domain. The partial derivation $\partial / \partial Y$ induces a derivation $D$ of $A$ over $k$. If $D$ were integrable to $\underline{D}=\left(1, D_{1}, D_{2}, \cdots\right) \in \operatorname{HS}(A)$ with $D_{1}=D$, then we should have

$$
0=D_{p}\left(y^{p}-x^{p}-x^{p+1}\right)=D(y)^{p}-D(x)^{p}-\sum_{i=0}^{p} D_{i}\left(x^{p}\right) D_{p-i}(x)=1-x^{p} D_{p}(x) .
$$

Therefore $D_{p}(x)=1 / x^{p}$. But $1 / x^{p}$ is not in $A$. Hence $D$ is not integrable.

Example 4. Let $B$ be a ring and $A=B[[X]]$ be the formal power series ring over $B$. Let $t$ be another indeterminate. Then the map $f(X)$ $\mapsto f(X+t)$ defines an iterative differentiation of $A$. Similarly for $A[X]$.

\section{§2. Seidenberg Theorems}

Let $A$ be a ring, $I$ an ideal of $A$ and $\underline{D}=\left(1, D_{1}, D_{2}, \cdots\right) \in \operatorname{HS}(A)$. The ideal $I$ is said to be $\underline{D}$-invariant (or invariant under $\underline{D}$ ) if $D_{i}(I) \subseteq I$ for all $i$. When this is the case, the differentiation $\underline{D}$ induces a differentiation of $A / I$. Recall that an ideal of $A$ is called a differential ideal if all derivations of $A$ map the ideal into itself. We shall say that the ideal $I$ is a HS-ideal (resp. $\mathrm{HS}_{k}$-ideal) if it is invariant under all differentiations in $\mathrm{HS}(A)$ (resp. $\mathrm{HS}_{k}(A)$ ). If $A$ contains $\mathbf{Q}$, then the differential ideals and the HS-ideals are the same (this can be seen using Remark 2 of $\S 1$.)

Theorem 1. Let $A$ be a ring, $I$ an ideal of $A$ and $t$ an indeterminate over $A$; put $A^{*}=A[[t]]$ and $I^{*}=I[[t]]$. Let $D \in \operatorname{HS}(A)$. Then $I$ is $\underline{D}$ invariant if and only if the automorphism $E_{t}$ of $A^{*}$ associated to $\underline{D}$ maps $I^{*}$ onto itself: $E_{t}\left(I^{*}\right)=I^{*}$. 
Proof. If $D_{i}(I) \subseteq I$ for all $i$, we have $E_{t}\left(I^{*}\right) \subseteq I^{*}$. It is easy to see that $\left(E_{t}\right)^{-1}(a)=\sum t^{n} D_{n}^{\prime}(a)$ where $D_{n}^{\prime}$ is a polynomial in $D_{1}, \cdots, D_{n}$. Therefore we have $\left(E_{t}\right)^{-1}\left(I^{*}\right) \subseteq I^{*}$ also. Thus $E_{t}\left(I^{*}\right)=I^{*}$. The converse is obvious.

Theorem 2. Let $A$ be a noetherian ring and $P \in \operatorname{Ass}(A)$. Then $P$ is an HS-ideal, and consequently there are canonical maps

$$
\mathrm{HS}(A) \longrightarrow \mathrm{HS}(A / P), \quad \operatorname{Ider}(A) \longrightarrow \operatorname{Ider}(A / P) \text {. }
$$

Proof. We give only a sketch of Seidenberg's proof in [19] pp. 23-24. If (0) $=q_{1} \cap \cdots \cap q_{r}$ is an irredundant primary decomposition in $A$ and if $p_{i}$ is the associated prime ideal of $q_{i}$, then $(0)=q_{1}^{*} \cap \cdots \cap q_{r}^{*}$ is an irredundant primary decomposition in $A^{*}$ and $p_{i}^{*}$ is the associated prime ideal of $q_{i}^{*}$. Thus any automorphism $E$ of $A^{*}$ induces a permutation of Ass $\left(A^{*}\right)=\left\{p_{1}^{*}, \cdots, p_{r}^{*}\right\}$. If $E$ corresponds to a differentiation then from $E\left(p_{i}^{*}\right)=p_{j}^{*}$ it follows that $p_{i} \subseteq p_{j}$. Considering $E^{-1}$ we get $p_{i}=p_{j}$, or what amounts to the same, $p_{i}^{*}=p_{j}^{*}$. By the preceding theorem this means that $p_{1}, \cdots, p_{r}$ are HS-ideals.

Remark 3. Example 1 shows that $P$ is not necessarily a differential ideal.

Theorem 3. Let $A$ be a noetherian integral domain and $A^{\prime}$ be its derived normal ring. Then any differentiation of $A$ extends to $A^{\prime}$, and consequently there are canonical mappings

$$
\mathrm{HS}(A) \longrightarrow \mathrm{HS}\left(A^{\prime}\right), \quad \operatorname{Ider}(A) \longrightarrow \operatorname{Ider}\left(A^{\prime}\right) \text {. }
$$

Proof. Let $K$ denote the quotient field of $A$, let $\underline{D} \in \operatorname{HS}(A)$ and let $E: A \rightarrow A[[t]]$ denote the corresponding homomorphism. We know that $\underline{D}$ and $E$ can be extended uniquely to $K$; we denote the extensions by the same letters $\underline{D}$ and $E$. Then we have to show: $E\left(A^{\prime}\right) \subseteq A^{\prime}[[t]]$.

It is well known that $A[[t]]$ is normal if $A$ is a noetherian normal ring. In the present case the ring $A^{\prime}$ is not necessarily noetherian, but still it is a Krull ring (cf. Nagata, Local Rings, p. 118), therefore an intersection of discrete valuation rings: $A^{\prime}=\bigcap_{\alpha} V_{\alpha}$. Then $A^{\prime}[[t]]=\bigcap_{\alpha} V_{\alpha}[[t]]$, and each $V_{\alpha}[[t]]$ is normal. Therefore $A^{\prime}[[t]]$ is also normal. Let $a^{\prime} \in A^{\prime}$, $a^{\prime}=u / v, u \in A, v \in A$. Then $E\left(a^{\prime}\right)=E(u) / E(v)$ belongs to the quotient field of $A[[t]]$. Moreover, since $a^{\prime}$ is integral over $A, E\left(a^{\prime}\right)$ is integral over $A[[t]]$, hence a fortiori over $A^{\prime}[[t]]$. Therefore $E\left(a^{\prime}\right) \in A^{\prime}[[t]] . \quad$ Q.E.D. 
Remark 4. If $A^{\prime}$ is finite over $A$ then $A^{\prime}[[t]]$ is finite over $A[[t]]$ and is equal to the derived normal ring of $A[[t]]$.

Remark 5. Example 3 of $\S 1$, which is also due to Seidenberg, shows that a non-integrable derivation of $A$ does not necessarily extend to $A^{\prime}$.

Corollary. Let $A, A^{\prime}$ be as in Th. 3 and $c$ be the conductor of $A$ (i.e. $\mathfrak{c}=\left\{a \in A \mid a A^{\prime} \subseteq A\right\}$ ). Then $\mathfrak{c}$ is an HS-ideal.

Proof. Let $a \in \mathfrak{c}, x \in A^{\prime}, \underline{D}=\left(1, D_{1}, D_{2}, \cdots\right) \in \operatorname{HS}(A)$. Then $a x \in A$ and so $D_{n}(a x)=D_{n}(a) x+D_{n-1}(a) D_{1}(x)+\cdots+a D_{n}(x) \in A$. We prove $D_{n}(a) \in \mathfrak{c}$ by induction on $n$. Suppose $D_{i}(a) \in \mathfrak{c}$ for $i<n$. Since $D_{i}(x) \in A^{\prime}$ for all $i$, we have $D_{n}(a) x \in A$. As $x$ is an arbitrary element of $A^{\prime}$ this means that $D_{n}(a) \in \mathrm{c}$.

Theorem 4. Let $A$ be an excellent ring, $I$ be the largest ideal which defines Sing $(A)$ and $P$ be the generic point of an irreducible component of Sing $(A)$. Then $I$ and $P$ are HS-ideals.

Proof. Since $P$ is an associated prime of $I$, if $I$ is an HS-ideal then $P$ is so by Th. 2. Thus it suffices to prove that $I^{*}$ is invariant under any automorphism of $A^{*}=A[[t]]$. Now $A^{*}$ is the $t$-adic completion of $A[t]$. Since $A[t]$ is excellent the canonical homomorphism $A[t] \rightarrow A^{*}$ is regular by a well-known theorem of Grothendieck (cf. [EGA IV-2] 7.8 .3 (v) or [11] Th. 79). On the other hand it is obvious that the canonical map $A \rightarrow A[t]$ is regular (for any $A$ ). Therefore $A \rightarrow A^{*}$ is regular. It follows that $I^{*}$ defines Sing $\left(A^{*}\right)$. Since $I$ is reduced (i.e. an intersection of prime ideals), so is $I^{*}$. Thus $I^{*}$ is the largest ideal which defines $\operatorname{Sing}\left(A^{*}\right)$, and, as such, is invariant under any automorphism of $A^{*}$.

Q.E.D.

Remark 6. Similarly, the largest ideal which defines the set $\{\mathfrak{p} \in$ $\operatorname{Spec}(A) \mid A_{p}$ is not $\left.Q\right\}$, where $Q$ denotes the property normal, CohenMacaulay, Gorenstein (cf. [21]), or complete intersection (cf. [3]), is an HS-ideal.

Let $k$ be a field and $A=k\left[x_{1}, \cdots, x_{n}\right]$ be a finitely generated $k$-algebra. Put $R=k\left[X_{1}, \cdots, X_{n}\right]$, and write $A=R / I$, where $I$ is the kernel of the $k$-algebra homomorphism $R \rightarrow A$ which sends $X_{i}$ to $x_{i}$. Let $f_{1}, \cdots, f_{s}$ be a system of generators of $I$. We write $\partial f / \partial x_{i}$ for $\partial f / \partial X_{i} \bmod I$. Consider the Jacobian matrix $(\partial f / \partial x)=\left(\partial f_{i} / \partial x_{j}\right)_{1 \leqslant i \leqslant s, 1 \leqslant j \leqslant n}$. Let $\nu$ be an integer, $0 \leqslant \nu<n$. The ideal of $A$ generated by the $(n-\nu) \times(n-\nu)$ minors of $(\partial f / \partial x)$ will 
be called the $\nu$-th Jacobian ideal of $A$ and will be denoted by $J_{\nu}(A)$ or simply by $J_{\nu}$. We put $J_{n}=J_{n+1}=\cdots=A$. Then we have $J_{0} \subseteq J_{1} \subseteq J_{2}$ $\subseteq \cdots$ Lipman [9] calls the first non-zero $J_{\nu}$ the Jacobian ideal of $A$. When $k$ is a perfect field and $A$ is an integral domain of dimension $d$, it is known that the matrix ( $\partial f / \partial x)$ has rank $n-d$ ([AG] pp. 32-33). Therefore $J_{d}$ is the Jacobian ideal of $A$ in this case.

The exact sequence (cf. [11] Th. 58)

$$
I / I^{2} \longrightarrow \Omega_{R / k} \otimes_{R} A=A d X_{1} \oplus \cdots \oplus A d X_{n} \longrightarrow \Omega_{A / k} \longrightarrow 0
$$

shows that $J_{\nu}$ is the $\nu$-th Fitting invariant of $\Omega_{A / k}$ (cf. [15]). Therefore the ideals $J_{\nu}$ are invariants of the $k$-algebra $A$, independent of the representation $A=R / I$ and of the choice of the generators $f_{1}, \cdots, f_{s}$ of $I$. We will state the invariance more precisely in the following lemma.

LEMma 1. The ideals $J_{\nu}$ are left fixed by all automorphisms of the $k$ algebra $A$.

Proof. Let $\sigma$ be an automorphism of the $k$-algebra $A$, and $M$ be an $A$-module. The $A$-module structure on $M$ is defined by a $k$-algebra homomorphism $\psi: A \rightarrow \operatorname{End}_{k}(M)$. We define a new $A$-module structure on $M$ by $\psi \circ \sigma$, and denote the new $A$-module by $M_{\sigma}$. Thus, $a x$ in $M_{\sigma}=a^{\sigma} x$ in $M(a \in A, x \in M)$. If $D: A \rightarrow M$ is a $k$-derivation, then $D \circ \sigma$ is a $k$-derivation of $A$ into $M_{\sigma}$. Call it $D^{\sigma}$.

$$
\begin{aligned}
D^{\sigma}(a b)=D\left(a^{\sigma} b^{\sigma}\right) & =a^{\sigma} D\left(b^{\sigma}\right)+b^{\sigma} D\left(a^{\sigma}\right) & & \text { in } M \\
& =a D^{\sigma}(b)+b D^{\sigma}(a) & & \text { in } M_{\sigma} .
\end{aligned}
$$

Let $\Omega_{A / k}=\sum_{1}^{n} A d x_{i} . \quad \sum a_{i} d x_{i}=0$ means that $\sum a_{i} D\left(x_{i}\right)=0$ holds for every $A$-module $M$ and for every derivation $D: A \rightarrow M$. Then $\sum a_{i} D^{\circ}\left(x_{i}\right)=0$ in $M_{\sigma}$, i.e. $\sum a_{i}^{\sigma} D\left(x_{i}^{\sigma}\right)=0$ in $M$. Therefore we have $\sum a_{i}^{\sigma} d x_{i}^{\sigma}=0$. Thus, by putting

$$
\left(\sum a_{i} d x_{i}\right)^{\sigma}=\sum a_{i}^{\sigma} d x_{i}^{\sigma}
$$

we can define an automorphism of the $k$-module $\Omega_{A / k}$ such that

$$
(a \omega)^{\sigma}=a^{\sigma} \omega^{\sigma} \quad\left(a \in A, \omega \in \Omega_{A / k}\right) .
$$

If $d x_{1}, \cdots, d x_{n}$ generate $\Omega_{A / k}$, then $d x_{1}^{\sigma}, \cdots, d x_{n}^{\sigma}$ also generate $\Omega_{A / k}$. Moreover, the $\sigma$-image of a relation matrix of $d x_{1}, \cdots, d x_{n}$ is a relation matrix of $d x_{1}^{\sigma}, \cdots, d x_{n}^{\sigma}$. By the independence of Fitting ideals on the choice of generators of the $A$-module, our lemma is now obvious. 
Let $B$ be a $k$-algebra. The module $\Omega_{B / k}$ represents the functor $M \rightarrow$ $\operatorname{Der}_{k}(B, M)$ on the category of all $B$-modules. If the restriction of this functor to the category of finite $B$-modules is representable, i.e. if there exist a finite $B$-module $M_{0}$ and a $k$-derivation $d_{0}: B \rightarrow M_{0}$ with the universal mapping property for the $k$-derivations of $B$ into finite $B$-modules, then $M_{0}$ is called the universal finite module of differentials of $B$ over $k$ and is denoted by $D_{k}(B)$, cf. [17] or [22]. The following lemmas can be easily proved from the definition.

Lemma 2. Let $B$ be a noetherian k-algebra such that $D_{k}(B)$ exists. Then $D_{k}\left(B^{*}\right)$ also exists (where $B^{*}=B[[t]]$ ), and we have

$$
D_{k}\left(B^{*}\right)=\left(D_{k}(B) \otimes_{B} B^{*}\right) \oplus B^{*} d t .
$$

Lemma 3. Let $R$ be a noetherian k-algebra, $I$ an ideal of $R$ and $B=$ $R / I$. Suppose $D_{k}(R)$ exists. Then $D_{k}(B)$ also exists, and we have an exact sequence

$$
I / I^{2} \longrightarrow D_{k}(R) \otimes_{R} B \longrightarrow D_{k}(B) \longrightarrow 0 \text {. }
$$

(cf. [22].)

Returning to the situation $R=k\left[X_{1}, \cdots, X_{n}\right], I=\left(f_{1}, \cdots, f_{s}\right)$ and $A$ $=R / I$, we have

$$
A^{*}=R^{*} / I^{*}, \quad I^{*}=\sum_{1}^{n} f_{i} R^{*}
$$

and the sequence

$$
I^{*} / I^{* 2} \longrightarrow D_{k}\left(R^{*}\right) \otimes_{R^{*}} A^{*} \longrightarrow D_{k}\left(A^{*}\right) \longrightarrow 0
$$

is exact. Moreover, $D_{k}\left(R^{*}\right) \otimes_{R^{*}} A^{*}=\left(D_{k}(R) \otimes_{R} A^{*}\right) \oplus A^{*} d t$ is a free $A^{*}$. module with basis $d X_{1}, \cdots, d X_{n}, d t$. Therefore $J_{\nu} A^{*}$ is the $(\nu+1)$ st Fitting invariant of $D_{k}\left(A^{*}\right)$, and proof of Lemma 1 can be applied, mutatis mutandis, to show that $J_{\nu} A^{*}$ is invariant under all $k$-algebra automorphisms of $A^{*}$. This proves the following theorem.

Theorem 5. Let $k$ be a field and $A$ be a k-algebra of finite type. Then the ideals $J_{\nu}$ are $\mathrm{HS}_{k}$-ideals.

EXAMPLE 5. Let $k$ be a field of characteristic $p \geqslant 0$ and let $A=$ $k[x, y]=k[X, Y] /\left(Y^{2}-X^{3}\right)$. The derived normal ring $A^{\prime}$ is $k[u]$ where $u$ $=y / x$, and we have $x=u^{2}, y=u^{3}$. The conductor is $x A^{\prime}=(x, y) A$, which is also the largest ideal that defines $\operatorname{Sing}(A)$. Put $D_{0}=d / d u \in \operatorname{Der}_{k}\left(A^{\prime}\right)$. 
The derivation $u D_{0}$ induces an integrable derivation $D_{1}$ of $A$ because $E_{t}: A^{\prime} \rightarrow A^{\prime}[[t]]$ defined by $E_{t}(u)=u(1+t)$ maps $x=u^{2}$ and $y=u^{3}$ into $A[[t]]$. Similarly $u^{2} D_{0}$ induces an integrable derivation $D_{2}$ of $A$. Let $D$ $\in \operatorname{Ider}_{k}(A)$. Then $D \in \operatorname{Ider}_{k}\left(A^{\prime}\right)=A^{\prime} D_{0}$. If $p \neq 2$ then $D_{0}(x)=2 u \notin A$ and so $D_{0} \notin \operatorname{Der}_{k}(A)$. If $p=2$ then for any element $f$ in $k[u]$ we have $\left(u+t+f t^{2}\right)^{3}=u^{3}+3 u^{2} t+3\left(u+u^{2} f\right) t^{2}+\cdots$, and $u+u^{2} f \notin A$. Thus $D_{0} \notin$ $\operatorname{Ider}_{k}(A)$ in all cases. Therefore we have $\operatorname{Ider}_{k}(A)=A D_{1}+A D_{2}$. When $p \neq 2,3$ it is easy to see that $\operatorname{Der}_{k}(A)=A D_{1}+A D_{2}=\operatorname{Ider}_{k}(A)$.

If $p=2$ then the Jacobian ideal of $A$ is $x^{2} A$. The partial derivation $\partial / \partial Y$ of $k[X, Y]$ induces a derivation $D_{3}$ on $A$, and $D_{0}=x D_{3}$. We have $\operatorname{Der}_{k}(A)=A D_{3}, \operatorname{Ider}_{k}(A)=A D_{1}+A D_{2}=A y D_{3}+A x^{2} D_{3}$. The derivation $D_{0}$ maps $x^{2} A$ and $(x, y) A$ into themselves, but it is not integrable as we have already seen.

If $p=3$ the partial derivation $\partial / \partial X$ induces a derivation $D_{4}$ on $A$. We have $\operatorname{Der}_{k}(A)=A D_{4}, \operatorname{Ider}_{k}(A)=A x D_{4}+A y D_{4}$.

\section{§3. Integrability and smoothness}

The theorems of the preceding section give various necessary conditions for a derivation to be integrable. In this section we will consider sufficient conditions of integrability.

Let $k$ be a ring and $A$ a $k$-algebra. To give a derivation $D \in \operatorname{Der}_{k}(A)$ is to give a $k$-algebra homomorphism $\phi_{1}: A \rightarrow A[t] /\left(t^{2}\right)$ such that $\phi_{1}(a) \equiv a$ $\bmod t$. Saying that $D$ is integrable (over $k$ ) is equivalent to saying that $\phi_{1}$ can be lifted to a $k$-algebra homomorphism $E: A \rightarrow A[[t]]$, and since $A[[t]]=\lim A[t] /\left(t^{n}\right)$ it suffices to find, step by step, $k$-algebra homomorphisms $\phi_{n}: A \rightarrow A[t] /\left(t^{n+1}\right)$ such that $\phi_{n-1}(a)=\phi_{n}(a) \bmod t^{n}$. Such lifting is always possible if $A$ is a smooth $k$-algebra in the sense of [11] (i.e. formally smooth with respect to the discrete topology in the sense of EGA, or 0smooth in the sense of André [1].)

TheоRem 6. Let $k$ be a field and $K$ be a separable extension field of $k$. Then $K$ is a smooth k-algebra. Consequently, every derivation of $K$ over $k$ is integrable over $k$.

Proof. The smoothness is well known, cf. [5], [11]. Actually, one can say more: Let $B$ be a differential basis of $K$ over $k$. Then $k(B)$ is a purely transcendental extension of $k$, and $K$ is formally etale over $k(B)$. (Cf. [10, Th. 2].) 
Corollary. Let $K$ be a field. Then any derivation of $K$ is integrable.

Proof. Put $k=$ the prime field in $K$ in the theorem.

Lemma 4. Let $A$ be a ring of characteristic $p$, and $D$ be a derivation of $A$ with $D^{p}=0$. Put $A_{0}=\{a \in A \mid D a=0\}$. If $x \in A$ satisfies $D x=1$, then $A$ is a free $A_{0}$-module with $1, x, x^{2}, \cdots, x^{p-1}$ as a basis.

Proof. Put $A_{i}=\left\{a \in A \mid D^{i+1} a=0\right\}$ for $0 \leqslant i<p$. By the assumption $D^{p}=0$ we have $A_{p-1}=A$. We will prove

$$
A_{i}=A_{0}+A_{0} x+\cdots+A_{0} x^{i}
$$

by induction on $i$. For $i=0$ there is nothing to prove. Let $D^{i+1} a=0$. Then $D^{i} a \in A_{0}$, and if we put $b=a-(i !)^{-1} x^{i} D^{i} a$, then $D^{i} b=0$, i.e. $b \in A_{i-1}$ $=A_{0}+A_{0} x+\cdots+A_{0} x^{i-1}$. Thus $a \in A_{0}+A_{0} x+\cdots+A_{0} x^{i}$, as wanted. The linear independence of $1, x, \cdots, x^{p-1}$ over $A_{0}$ is obvious.

Theorem 7. Let $K$ be a separable extension field of a field $k$ of characteristic $p$. Let $D \in \operatorname{Der}_{k}(K)$. Then $D$ is strongly integrable over $k$ iff $D^{p}=0$.

Proof. We have already seen the necessity. To prove the sufficiency, we may assume $D \neq 0, D^{p}=0$. Take $y \in K$ with $D y \neq 0$. Then there exists a positive integer $i<p$ such that $D^{i} y \neq 0, D^{i+1} y=0$. Put $x=$ $D^{i-1} y / D^{i} y$. Then $D x=1$. Therefore, putting $K_{0}=\{a \in K \mid D a=0\}$ we have $K=K_{0}(x)$ and $\left[K: K_{0}\right]=p$ by Lemma 4 . The separability of $K / k$ implies that $K^{p}$ and $k$ are linearly disjoint over $k^{p}$. Suppose $x^{p} \in K_{0}^{p} k$. Then we can write $x^{p}=\sum_{i=1}^{r} y_{i}^{p} c_{i}$, where $y_{i} \in K_{0}, c_{i} \in k$ and $y_{1}^{p}, \cdots, y_{r}^{p}$ are linearly independent over $k^{p}$. Then $y_{1}, \cdots, y_{r}$ are linearly independent over $k$, and since $x \notin K_{0}$ and $k \subset K_{0}$ we see that $x, y_{1}, \cdots, y_{r}$ are also linearly independent over $k$. Therefore $x^{p}, y_{1}^{p}, \cdots, y_{r}^{p}$ must be linearly independent over $k^{p}$, hence over $k$ by the linear disjointness. But this contradicts our assumption $x^{p}=\sum y_{i}^{p} c_{i}$. Therefore $x^{p} \notin K_{0}^{p} k$, and so there exists a $p$-basis $B_{0}$ of $K_{0} / k$ containing $x^{p}$ as a member. Put

$$
B=\left(B_{0}-\left\{x^{p}\right\}\right) \cup\{x\} .
$$

Then, putting $y=x^{p}$ and $I=\left(X^{p}-y\right) K_{0}[X]$, we have $K=K_{0}[X] / I$. The exact sequence

$$
I / I^{2} \longrightarrow \Omega_{K_{0}[X] / k} \otimes K=\left(\Omega_{K_{0} / k} \otimes K\right) \oplus K d X \longrightarrow \Omega_{K / k} \longrightarrow 0
$$


shows

$$
\Omega_{K / k} \simeq\left(\left(\Omega_{K_{0} / k} \otimes_{K_{0}} K\right) / K d y\right) \oplus K d x .
$$

This means that $B$ is a $p$-basis of $K / k$. Put $k^{\prime}=k\left(B_{0}-\left\{x^{p}\right\}\right)$. Then $x$ is transendental over $k^{\prime}$ (cf. [10, Th. 1]), hence we can define a homomorphism of $k^{\prime}$-algebras

$$
E_{t}: k^{\prime}(x) \longrightarrow k^{\prime}(x)[[t]]
$$

by $E_{t}(x)=x+t$. Since $K$ is formally etale over $k^{\prime}(x)=k(B)$, it follows from the diagram

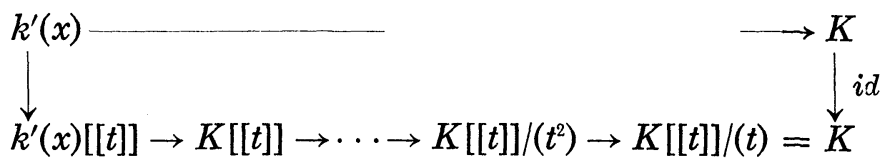

that $E_{t}$ can be uniquely extended to a homomorphism of $k^{\prime}$-algebras

$$
E_{t}: K \longrightarrow K[[t]]
$$

Consider the diagram

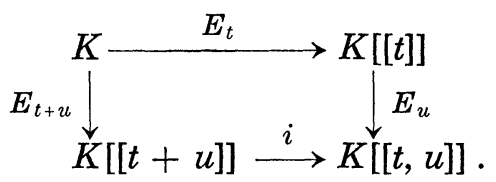

We have $E_{u} E_{t}(a) \equiv a \equiv E_{t+u}(a) \bmod (t, u)$ for all $a \in K$ and $E_{u} \circ E_{t}=i \circ E_{t+u}$ on $k^{\prime}(x)$. Hence the diagram commutes by the formal etaleness of $K / k^{\prime}(x)$. Therefore $E_{t}$ determines an iterative differentiation $\underline{D}=\left(1, D_{1}, D_{2}, \cdots\right)$ of $K$ over $k^{\prime}$ such that $D_{1}(x)=1=D(x), D_{i}(x)=0(i>1)$. Since $D_{1}(\alpha)=$ $0=D(\alpha)$ for $\alpha \in K^{p} k^{\prime}=K_{0}$, we have $D_{1}=D$. Q.D.

Resuming our general discussion at the beginning of this section, we put $A_{n}=A[t] /\left(t^{n+1}\right)$ and consider the extension of $k$-algebras

$$
0 \longrightarrow N \longrightarrow A_{n} \stackrel{\pi}{\longrightarrow} A_{n-1} \longrightarrow 0,
$$

where $N=A t^{n}$ is an ideal of square zero in $A_{n}$ and $N \simeq A$ as $A$-module. The pull-back of (3.1) by $\phi_{n-1}: A \rightarrow A_{n-1}$ is the extension

$$
0 \longrightarrow A \longrightarrow B \longrightarrow A \longrightarrow 0
$$

where $B$ is the fibre product of $A$ and $A_{n}$ over $A_{n-1}$ :

$$
B=\left\{(\alpha, a) \in A_{n} \times A \mid \pi(\alpha)=\phi_{n-1}(a)\right\} .
$$


The extension (3.2) is trivial if and only if $\phi_{n-1}$ is liftable to $A \rightarrow A_{n}$. Thus the obstruction to lifting $\phi_{n-1}$ is the cohomology class represented by (3.2) in the group $H^{1}(k, A, A)$ of M. André. (Cf. [1] Chap. XVI. It coincides with the group $\operatorname{Exalcom}_{k}(A, A)$ of EGA.) Therefore we have

TheOREM 8. Let $k$ be a ring and $A$ be a k-algebra. If

$$
H^{1}(k, A, A)=0,
$$

then every derivation $D$ of $A$ over $k$ is integrable over $k$.

Remark 7. As a matter of fact the extensions (3.1), (3.2) are Hochschild extensions, and so the obstruction class lies in the subgroup $H_{k}^{2}(A, A)^{s}$ of $\operatorname{Exalcom}_{k}(A, A)$, cf. [5] p. 65. But we will not discuss this group here.

We will apply Th. 8 to regular local rings of characteristic $p$. Let $(A, m, K)$ be a regular local ring, and $k$ be a field of characteristic $p$ contained in $A$. If the residue field $K$ is separable over $k$ then $A$ is formally smooth (with respect to the $m$-adic topology) over $k$, but not conversely.

Formal smoothness is equivalent to $H^{1}(k, A, K)=0$, and then $H^{1}(k$, $A, M)=0$ for all $A$-modules $M$ which satisfy $m^{\nu} M=0$ for some $\nu$. Smoothness is equivalent to $H^{1}(k, A, M)=0$ for all $A$-modules $M$. ([1] p. 223 Prop. 17, p. 222 Def. 14.) Also the following lemma is known.

Lemma 5. Let $(A, m, K)$ be a noetherian local ring containing a field k. Assume that $A$ is formally smooth (with respect to the maximal ideal) over $k$. Then:

i) for any prime ideal $P$ of $A$ the local ring $A_{P}$ is formally smooth over $k$,

ii) $H_{i}(k, A, M)=0$ for all A-modules $M$ and for all $i>0$,

iii) $H_{0}(k, A, A)=\Omega_{A / k}$ is A-flat,

iv) $H^{i}(k, A, M)=\operatorname{Ext}_{A}^{i}\left(H_{0}(k, A, A), M\right)$ for all A-modules $M$ and for all $i \geqslant 0$.

Proof. i) Formal smoothness over $k$ is equivalent to geometric regularity over $k\left([5](22.5 .8)\right.$, [11] p. 279 Th. 93). If $k^{\prime}$ is a finite extension field of $k$, then $A_{P} \otimes_{k} k^{\prime}$ is a localization of $A \otimes_{k} k^{\prime}$. Therefore it is regular.

ii) follows from i) and [1] p. 331 Th. 30.

iii) and iv): By [1] p. 41 Lemma $19, H_{i}(k, A, A)=0(i>0)$ implies

$H_{i}(k, A, M)=\operatorname{Tor}_{i}^{A}\left(H_{0}(k, A, A), M\right), \quad H^{i}(k, A, M)=\operatorname{Ext}_{A}^{i}\left(H_{0}(k, A, A), M\right)$ 
for all $i \geqslant 0$. The first equation and ii) imply that $H_{0}(k, A, A)$ is $A$-flat.

TheOREM 9. Let $k$ be a field and $A$ be a noetherian local ring containing $k$. Assume that $A$ is formally smooth over $k$ and that $\Omega_{A / k}$ is a finite A-module. Then $A$ is smooth over $k$. Consequently, we have

$$
\operatorname{Der}_{k}(A)=\operatorname{Ider}_{k}(A) \text {. }
$$

Proof. The module of differentials $\Omega_{A / k}$ is finite by assumption and flat by Lemma 5. Hence it is free, and so $H^{1}(k, A, M)=\operatorname{Ext}_{A}^{1}\left(\Omega_{A / k}, M\right)=$ 0 for every $A$-module $M$. Therefore $A$ is smooth over $k$.

Remark 8. The finiteness of $\Omega_{A / k}$ holds in each of the following cases:

1) $A$ is a localization of a finitely generated $k$-algebra;

2) $\operatorname{char}(k)=p$ and $A$ is finite over $k\left[A^{p}\right]$.

The second case includes in particular $k\left[\left[X_{1}, \cdots, X_{n}\right]\right]$ with $\left[k: k^{p}\right]$ finite.

Theorem 10. If $A$ is a complete local ring formally smooth over a subfield $k$, then $H^{1}(k, A, M)=0$ for all finite A-module $M$. Consequently, we have

$$
\operatorname{Der}_{k}(A)=\operatorname{Ider}_{k}(A) .
$$

Proof. Consider an extension of $k$-algebras

$$
0 \longrightarrow N \longrightarrow B \stackrel{\alpha}{\longrightarrow} A \longrightarrow 0
$$

where $N$ is a finite $A$-module. Let $m$ denote the maximal ideal of $A$. The extension $0 \rightarrow N / m N \rightarrow B / m N \stackrel{\alpha_{1}}{\rightarrow} A \rightarrow 0$ splits because $N / m N$ is an $A / m$-module. Therefore there exists a $k$-algebra homomorphism $\phi_{1}: A \rightarrow$ $B / m N$ such that $\alpha_{1} \circ \phi_{1}=$ identity. Using formal smoothness we can lift $\phi_{1}$ to $\phi_{2}, \phi_{3}, \cdots$, where $\phi_{i}: A \rightarrow B / m^{i} N$, successively, because the kernel of the natural map $B / m^{i+1} N \rightarrow B / m^{i} N$ is an $A / m$-module. Since $N$ is a finite $A$-module, it is $m$-adically complete and separated. It follows easily that $B$ is canonically isomorphic to $\lim B / m^{i} N$. Therefore we obtain a $k$-algebra homomorphism $\phi: A \rightarrow B$ by $\phi \leftarrow \lim \phi_{i}$. Since $\alpha=\alpha_{1} \circ p_{1}$ (where $p_{1}$ is the natural map $B \rightarrow B / m N)$ and $p_{1} \circ{ }^{\leftarrow}=\phi_{1}$, we get $\alpha \circ \phi=\alpha_{1} \circ p_{1} \circ \phi=\alpha_{1} \circ \phi_{1}=$ identity. Therefore every extension of $A$ by $N$ splits, or equivalently, $H^{1}(k, A, N)=0$.

Q.E.D.

The author does not know whether $H^{1}(k, A, A)$ is zero for every formally smooth local $k$-algebra $A$, nor whether $H^{1}(k, A, A)=0$ for a local 
$k$-algebra $A$ (essentially of finite type, say) implies that $A$ is regular. Of course the equality $\operatorname{Der}_{k}(A)=\operatorname{Ider}_{k}(A)$ may happen even if $H^{1}(k, A, A)$ $\neq 0$. But anyway normality of a local ring is not enough to guarantee the integrability of all derivations, as we see in the following example.

EXAMPLE 6 . Let $k$ be a field of characteristic 2 and consider

$$
A=k[x, y, z]_{(x, y, z)}, \quad x y=z^{2} .
$$

This is a local ring of dimension 2 , and since it is a complete intersection and has an isolated singular point, it is normal. The derivations $\partial / \partial Z$ and $X \partial / \partial X+Y \partial / \partial Y$ of $k[X, Y, Z]$ induce derivations $D_{1}, D_{2}$ of $A$. Suppose $D_{1}$ is integrable. Then there exist power series

$$
E_{t}(x)=x+t^{2} \xi_{2}+\cdots, E_{t}(y)=y+t^{2} \eta_{2}+\cdots, E_{t}(z)=z+t+t^{2} \zeta_{2}+\cdots
$$

$\left(\xi_{i}, \eta_{i}, \zeta_{i} \in A\right)$ such that

$$
\left(x+t^{2} \xi_{2}+\cdots\right)\left(y+t^{2} \eta_{2}+\cdots\right)=\left(z+t+t^{2} \zeta_{2}+\cdots\right)^{2} .
$$

Then $x \eta_{2}+y \xi_{2}=1$, hence $1 \in m_{A}$, contradiction. Therefore $D_{1}$ is not integrable. One can show that $x D_{1}, y D_{1}, z D_{1}+D_{2} \in \operatorname{Ider}_{k}(A)$. The $A$-module $\operatorname{Der}_{k}(A)$ is a free module generated by $D_{1}, D_{2}$.

We recall the famous Zariski-Lipman conjecture: Let $A$ be the local ring of a point of a variety over a field $k$ of characteristic zero. If $\operatorname{Der}_{k}(A)$ is free then $A$ is regular. Lipman [8] proved that $A$ is normal. The conjecture has been proved only in the case of a hypersurface by Scheja-Storch [17]. The above example shows that the conjecture does not hold in characteristic $p$. But if we modify the conjecture as follows, then it may be true:

ConJeCture. If $k$ is perfect, if $\operatorname{Der}_{k}(A)=\operatorname{Ider}_{k}(A)$ and if this module is $A$-free, then $A$ is regular.

\section{$\S 4$. Finitely generated $\boldsymbol{k}$-algebras}

Let $k$ be a perfect field and let

$$
A=k\left[x_{1}, \cdots, x_{n}\right]=k\left[X_{1}, \cdots, X_{n}\right] / P, \quad P=\left(f_{1}, \cdots, f_{s}\right)
$$

be an integral domain of dimension $n-r$. Let $J$ be the Jacobian ideal of $A$, i.e. the ideal generated by the $r \times r$ minors of the Jacobian matrix $(\partial f / \partial x)$. (Cf. § 2.) We have seen that $D \in \operatorname{Ider}_{k}(A)$ implies $D(J) \subset J$. The converse is false, but we have the following theorem. 
Theorem 11. If $D \in \operatorname{Der}_{k}(A)$ and $D(A) \subset J$, then $D \in \operatorname{Ider}_{k}(A)$.

CoRollary 1. If $\Delta$ is a non-zero $r \times r$ minor of $(\partial f / \partial x)$, then

$$
\Delta \operatorname{Der}_{k}(A) \subset \operatorname{Ider}_{k}(A) \text {. }
$$

Consequently, we have

$$
\operatorname{rank} \operatorname{Der}_{k}(A)=\operatorname{rank} \operatorname{Ider}_{k}(A),
$$

where rank $M$ for an A-module $M$ means the maximal number of linearly independent elements in $M$.

Proof of Th. 11. Put $D\left(x_{i}\right)=\xi_{1 i}(\in J), \xi_{1}=\left(\xi_{11}, \cdots, \xi_{1 n}\right)$. Then we have

$$
f_{\alpha}\left(x+t \xi_{1}\right) \equiv 0 \bmod t^{2}, \quad 1 \leqslant \alpha \leqslant s .
$$

By induction, suppose that, for some $\nu>1$, we have found $\xi_{\mu j} \in J(1 \leqslant \mu<\nu$, $1 \leqslant j \leqslant n$ ) such that

$$
f_{\alpha}\left(x+\sum_{\mu=1}^{\nu-1} t^{\mu} \xi_{\mu}\right) \equiv 0 \bmod t^{\nu}, \quad 1 \leqslant \alpha \leqslant s .
$$

Then we can write

$$
f_{\alpha}\left(x+\sum_{1}^{\nu-1} t^{\mu} \xi_{\mu}\right) \equiv t^{\nu} F_{\alpha}(x) \bmod t^{\nu+1}, \quad 1 \leqslant \alpha \leqslant s .
$$

Then the $F_{a}(x)$ 's are linear combinations, with coefficients in $A$, of monomials of the form $\xi_{\mu_{1} j_{1}} \xi_{\mu_{2} j_{2}} \cdots \xi_{\mu_{q} j_{q}}, \mu_{1}+\mu_{2}+\cdots+\mu_{q}=\nu$. Since $\mu_{i}<\nu$ we have $q \geqslant 2$. Therefore $F_{\alpha}(x) \in J^{2}$. If $\xi_{\nu 1}, \xi_{\nu 2}, \cdots, \xi_{\nu n}$ are elements of $A$ we have

$$
f_{\alpha}\left(x+\sum_{1}^{\nu} t^{\mu} \xi_{\mu}\right) \equiv t^{\nu}\left[F_{\alpha}(x)+\sum_{j=1}^{n}\left(\partial f_{\alpha} / \partial x_{j}\right) \xi_{\nu j}\right] \bmod t^{\nu+1}, \quad 1 \leqslant \alpha \leqslant s .
$$

Therefore, if we can find $\xi_{\nu j} \in J(1 \leqslant j \leqslant n)$ which satisfy

$$
F_{\alpha}(x)+\sum_{j}\left(\partial f_{\alpha} / \partial x_{j}\right) \xi_{\nu j}=0 \quad(1 \leqslant \alpha \leqslant s)
$$

then we can continue the induction and we are done.

Let $\Delta_{1}, \cdots, \Delta_{a}$ be the non-zero $r \times r$ minors of the Jacobian matrix $(\partial f / \partial x)$. We may suppose that the first $r$ rows of the matrix $(\partial f / \partial x)$ are linearly independent. Put $k\left[X_{1}, \cdots, X_{n}\right]=R$. The local ring $R_{P}$ is regular of dimension $r$, and the map $\psi: R_{P} \rightarrow K^{n}(K=$ quotient field of $A$ ) defined by 


$$
\psi(f)=\left(\partial f / \partial x_{1}, \cdots, \partial f / \partial x_{n}\right)
$$

maps $P^{2} R_{P}$ to zero. Therefore $f_{1}, \cdots, f_{r}$ are linearly independent modulo $P^{2} R_{P}$, hence we have $P R_{P}=\left(f_{1}, \cdots, f_{r}\right) R_{P}$. Since $F_{\alpha}(x) \in J^{2}$ for all $\alpha$, we can write

$$
F_{i}(x)=\sum_{\lambda} \Delta_{\lambda} h_{\lambda \imath}(x), \quad h_{\lambda i} \in J(1 \leqslant i \leqslant r) .
$$

Let $f_{r+q}=\sum_{i=1}^{r} a_{q i} f_{i}, a_{q i}(X) \in R_{P}$. Then we have

$$
\partial f_{r+q} / \partial x_{j}=\sum a_{q i}(x) \partial f_{i} / \partial x_{j} .
$$

Moreover, we have $F_{r+q}(x)=\sum_{i} a_{q i}(x) F_{i}(x)$ because $f_{i}\left(x+\sum_{1}^{\nu-1} t^{\mu} \xi_{\mu}\right) \equiv t^{\nu} F_{i}(x)$ $\bmod t^{\nu+1}$. Thus, putting

$$
h_{\lambda, r+q}(x)=\sum_{1}^{r} a_{q i}(x) h_{\lambda i}(x)
$$

we see that (4.2) holds for $i=1, \cdots, s$.

Now fix an index $\lambda$ and consider the simultaneous equations

$$
\Delta_{\lambda} h_{\lambda i}(x)+\sum_{j=1}^{n}\left(\partial f_{\alpha} / \partial x_{j}\right) \xi_{j}^{(\lambda)}=0, \quad 1 \leqslant i \leqslant s .
$$

Let $\Gamma=\left\{i_{1}, \cdots, i_{r}\right\}$ denote the set of indices of the rows of $(\partial f / \partial x)$ which appear in $\Delta_{\lambda}$. These rows are linearly independent, and by (4.3) and (4.4) we have

$$
\operatorname{rank}\left(\begin{array}{ccc}
\partial f_{1} / \partial x_{1} \cdots \partial f_{1} / \partial x_{n} & h_{\lambda 1} \\
\vdots & \vdots & \\
\partial f_{s} / \partial x_{1} \cdots \partial f_{s} / \partial x_{n} & h_{\lambda s}
\end{array}\right)=\operatorname{rank}(\partial f / \partial x)=r
$$

Therefore, to solve (4.4) we have only to solve them for $i \in \Gamma$. We put $\xi_{j}^{(\lambda)}=0$ if the $j$-th columm of $(\partial f / \partial x)$ does not appear in $\Delta_{\lambda}$, and we find the other $\xi_{j}^{(\lambda)}$ by Cramer's rule. Since $h_{\lambda i}(x) \in J$ we have $\xi_{j}^{(\lambda)} \in J$. Then $\xi_{\lambda j}:=\sum_{l} \xi_{j}^{(\lambda)}$ satisfy (4.1).

Corollary 2. Let $k, A, J$ be as above and let $S$ be a multiplicative subset of $A$. Put $B=S^{-1} A$. Then $S^{-1} J=J B$ is the first non-zero Fitting ideal of $\Omega_{B / k}$, and if $D \in \operatorname{Der}_{k}(B)$ maps $B$ into $J B$ then $D \in \operatorname{Ider}_{k}(B)$.

Proof. There exists $a \in S$ such that $a D(A) \in J$. Then $a D \in \operatorname{Ider}_{k}(A)$, hence $D \in \operatorname{Ider}_{k}(B)$.

CoROLlaRY 3. Theorem 11 remains true if we replace the polynomial 
ring $k\left[X_{1}, \cdots, X_{n}\right]$ by the formal power series ring $k\left[\left[X_{1}, \cdots, X_{n}\right]\right]$.

Proof. The above proof of Th. 11 applies to this case as well.

Under the assumptions of Th. 11 we have $\operatorname{rank} \operatorname{Ider}_{k}(A)=\operatorname{rank} \operatorname{Der}_{k}(A)$ $=n-r=\operatorname{dim} A$. More generally, if $(A, m)$ is a noetherian local ring and $k$ is a quasi-coefficient field of $A$ (i.e. $k$ is a subfield of $A$ such that $A / m$ is formally etale over $k)$, then for each $P \in \operatorname{Ass}(\hat{A})$ we have rank $\operatorname{Ider}_{k}(A) \leqslant \operatorname{dim} \hat{A} / P$ (Mollinelli [12]), whereas $\operatorname{rank} \operatorname{Der}_{k}(A)$ can be bigger than $\operatorname{dim} A$. In the case when $k$ is imperfect Cor. 1 is false in general, as the following example shows.

ExAMPLE 7. Let $k$ be an imperfect field of characteristic $p>2$, and let $a, b \in k$ be such that $\left[k^{p}(a, b): k^{p}\right]=p^{2}$. Put $A=k[x, y]=k[X, Y] /$ $\left(X^{2 p}+a X^{p}+b Y^{p}\right)$. The partial derivations of $k[X, Y]$ induce derivations $D_{x}, D_{y}$ of $A$ over $k$, and we have $\operatorname{Der}_{k}(A)=A D_{x}+A D_{y}$. Suppose $u D_{x}$ $+v D_{y}$ is integrable, where $u=f(x, y)$ and $v=g(x, y)$. Considering the coefficient of $t^{p}$ in the relation $(x+t u+\cdots)^{2 p}+a(x+t u+\cdots)^{p}+b(y$ $+t v+\cdots)^{p}=0$ we get

$$
2 x^{p} u^{p}+a u^{p}+b v^{p}=0 .
$$

Therefore $2 X^{p} f(X, Y)^{p}+a f(X, Y)^{p}+b g(X, Y)^{p}=\left(X^{2 p}+a X^{p}+b Y^{p}\right) H(X, Y)$ for some $H(X, Y) \in k[X, Y]$. Applying derivations $D_{a}, D_{b}$ of $k$ such that $D_{a}(a)=1, D_{a}(b)=0, D_{b}(a)=0, D_{b}(b)=1$ to the last relation and substituting $x, y$ for $X, Y$ we get

$$
u^{p}=x^{p} w, \quad v^{p}=y^{p} w, \quad w=H(x, y) .
$$

Substituting them into $(*)$ we have $w=0$. Hence $u=v=0$. Thus $\operatorname{Ider}_{k}(A)=0$.

\section{REFERENCES}

[1] André, M., Homologie des algèbres commutatives, Springer, 1974.

[2] - Méthodes simpliciale en Algèbre Homologique et Algèbre Commutative, Springer Lecture Notes in Math., 32 (1967).

[3] Avramov, L. L., Flat morphisms of complete intersections, Soviet Math. Dokl., 16 (1975), 1413-1417.

[4] Brown, W. C., On the imbedding of derivations of finite rank into derivations of infinite rank, Osaka J. Math., 15 (1978), 381-389.

[ 5 ] Grothendieck, A., Éléments de Géométrie Algébrique, IV-1, Publ. I.H.E.S., no. 20, 1964.

[6 ] - ibid., IV-2, Publ. I.H.E.S., no. 24, 1965.

[ 7 ] Hasse, H. and Schmidt, F. K., Noch eine Begründung der Theorie der höheren 
Differentialquotienten in einem algebraischen Funktionenkorper einer Unbestimmten, J. reine u. angew. Math., 117 (1937).

[8] Lipman, J., Free derivation modules on algebraic varieties, Amer. J. Math., 87 (1965), 874-898.

[9] - On the Jacobian ideal of the module of differentials, Proc. Amer. Math. Soc., 21 (1969), 422-426.

[10] Matsumura, H., Quasi-coefficient rings of a local ring, Nagoya Math. J., 68 (1977), 123-130.

[11] Commutative Algebra, 2nd Ed. Benjamin, 1980.

[12] Molinelli, S., Sul modulo delle derivazioni integrabili in caratteristica positiva, Ann. Mat. Pura Appl., 121 (1979), 25-38.

[13] Nakai, Y., High order derivations I, Osaka J. Math., 7 (1970), 1-27.

[14] - On locally finite iterative higher derivations, ibid., 15 (1978), 655-662.

[15] Northcott, D. G., Finite Free Resolutions, Camb. Tracts in Math., 71, Camb. Univ. Press, 1976.

[16] Restuccia, G., Sul rango del modulo delle derivazioni di un anello in caratteristica diseguale, Rend. Sem. Mat. Univ. Politecn. Torino, 36 (1977-78), 449-462.

[17] Scheja-Storch, Differentielle Eigenschaften der Lokalisierungen analytiseher Algebren, Math. Ann., 197 (1972), 137-170.

[18] Seidenberg, A., Derivations and integral closure, Pacific J. Math., 16 (1966), 167-173.

[19] - Differential ideals in rings of finitely generated type, Amer. J. Math., 89 (1967), 22-42.

[20] - Differential ideals in complete local rings, ibid., 95 (1973), 52-58.

[21] Watanabe, K., Ishikawa, T., Tachibana, S. and Otsuka, K., On tensor products of Gorenstein rings, J. Math. Kyoto Univ., 9 (1969), 413-423.

[22] Yamauchi, N., On an algebra over a field with universal finite module of differentials, Nagoya Math. J., 83 (1981), 107-121.

[AG] Hartshorne, R., Algebraic Geometry, Springer, 1977.

Department of Mathematics

Nagoya University 